\title{
Damping evaluation of a ballistic foam: from tests to simulations
}

\author{
Jerome Mespoulet ${ }^{1 *}$, Hakim Abdulhamid ${ }^{1}$, and Paul Deconinck ${ }^{1}$ \\ ${ }^{1}$ Shock physics Department, THIOT INGENIERIE, 830 Route Nationale, 46130 Puybrun, France
}

\begin{abstract}
The perpetual evolution of soldiers light weight armors include now high technology ceramic, composite and polymeric in ballistic vest that are optimized by simulations. Knowledge of individual material response in the strain, strain rate regime closed to the threat stays mandatory and thus collecting parameters to fit material models guarantees reliable numerical investigations. Since 2015, THIOT INGENIERIE Shock Physics Laboratory has been selected by the French Defence procurement agency DGA-Land Systems to perform materials characterization in three main families of ballistic materials [1-2]. A coupled approach between laboratory experiments and numerical simulations has shown its relevance with ceramic and an Ultra High Molecular Weight PolyEthylene composite (UHMWPE). This paper presents succinctly the last part of those experimental investigations on a polymeric foam that is implemented on the soldier's chest [3]. The material behavior under dynamic loading has been first evaluated using Split Hokinson Pressure Bars (SHPB) up to $5000 \mathrm{~s}^{-1}$. Ballistic tests have been performed in a second time using Digital Image Correlation (DIC) with ultrahigh speed cameras at the back of the target plate to evaluate the damping behavior. Numerical simulations are under progress and the first results are promising.
\end{abstract}

\section{Introduction}

Reducing projectile kinetic energy is the main objective of soldier's light weight armor. Equation (1) illustrates this goal with Ep the initial kinetic energy of the projectile, $E_{A}$ the energy absorbed by the armor, $\mathrm{e}_{\mathrm{T}}$ the remaining transmitting energy per unit area to the foam and the soldier body and A the area on which this remaining energy is transmitted.

$$
E p=E_{A}+e_{T} * A
$$

\section{Context}

Fig. 1-A gives a description of a multi components armor and response of the front ceramic (2), the composite (3) and the back face mitigation foam (3) when impacted by a hard core bullet (1). The ceramic, as the first impacted layer, breaks the hard core and dwell soft sleeve of the projectile (B). The generated fragments, spread in a wider area, are captured and slow

*Corresponding author: mespoulet@thiot-ingenierie.com 
down by the composite (C) with the aid of the back face mitigation foam layer (D). This layer minimizes the Behind Armour Blunt Trauma (BABT) by distributing the remaining force on a larger area following a constant deceleration with a plateau stress like behaviour [3].
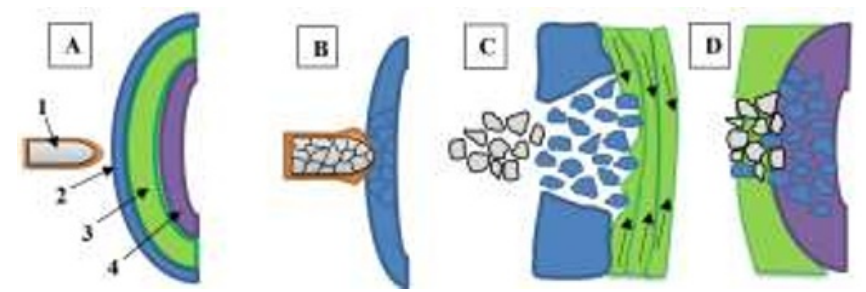

Fig. 1. Description of a cross-section of a hard core projectile / multi components soldier armour (a) and the various events from projectile impact to full arrest (b)-(d) [3]

\section{Material description}

Ballistic foam plates have been provided by DGA Land Systems [3]. High resolution SEM pictures done on the as-received material by DGA laboratory give cells diameters of $76 \mu \mathrm{m}+/-32 \mu \mathrm{m}$ in the 1-2 plane and $91+/-36 \mu \mathrm{m}$ in the 1-3 plan without skin effects: material is considered as isotropic based on small cell dimension compared to plate thickness [3]

\section{SHPB / Ballistic tests and simulations}

Positive strain rate sensitivity of the foam has been measured in SHPB tests as illustrated by a stress increase factor measured at $10 \%$ strain on the stress plateau for unconfined and confined tests (Fig2.-left). The factor is obtained by dividing the stress measured in the SHPB test by the value obtained in Quasi Static test. A dynamic amplification factor of 14 was obtained at $5000 \mathrm{~s}^{-1}$ for both configurations, the slope is higher in unconfined configuration.

The damping capacity of the polymeric foam has been evaluated through ballistic tests. The impact parameters (weights, dimensions and velocities of the projectile) were chosen to be representative of the actual threat, according to DGA Land Systems experience.

The impact configuration is proposed on Fig. 2-right: it consists in a $10 \mathrm{~mm}$ thick high strength aluminium witness plate firmly clamped on a target holder. Two metallic types of sphere, one small in steel and the other bigger in aluminium, have been used to represent the threat. Impact velocities for each type of sphere have been adjusted to be in the order of magnitude of the threat while ensuring a non-perforated configuration on the reference test that have been done with and without foams (metal - metal configuration). The back face of the target is monitored with a 3D Digital Image Correlation (DIC) to measure the out of plane deformation of the witness aluminium plate (Fig. 3.).
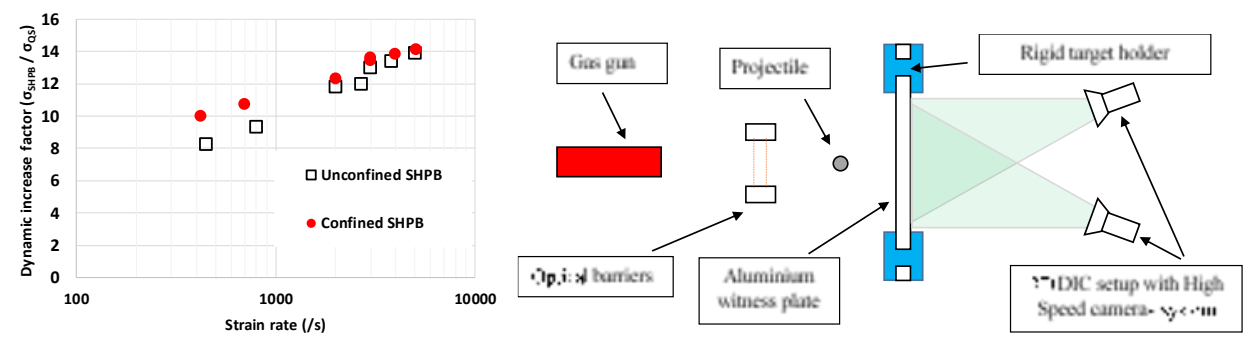

Fig. 2. Strain rate sensitivity (left) and ballistic set-up (right) 
Finite Element analysis of the ballistic tests were performed in LSDYNA ${ }^{\mathrm{TM}}$ (Fig. 3.middle) using $100 \%$ hexahedral solid elements. SHPB results were used to calibrate a tabulated foam material model. An additional curve with a highest dynamic increase factor at highest strain rate has been added with an erosion volumetric strain value to reproduce the deformation when element compression reached a too high value. Those assumptions are simple but indeed give good preliminary results as shown by Fig.3-right with the steel sphere [3]. The numerical model is able to correctly predict the out-of-plane displacement and dynamic response of the target (full results developed in [3]).
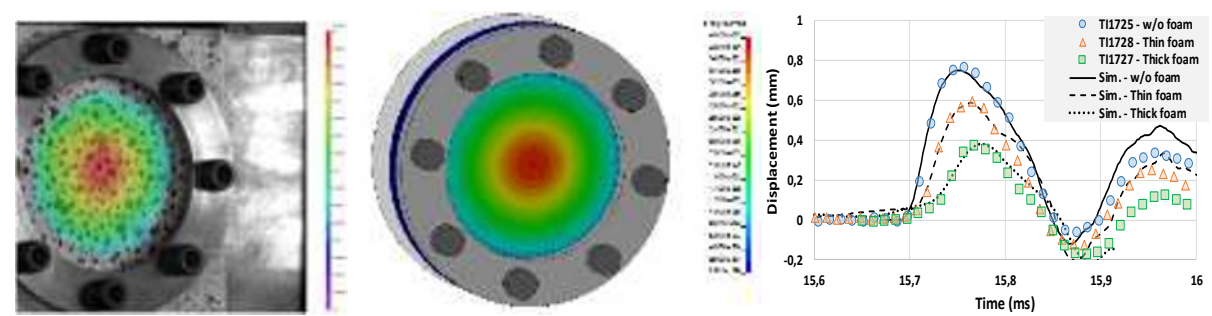

Fig. 3. Example of measured and calculated back face deformation [3]

\section{Conclusion}

This paper has presented a characterization study of a ballistic mitigation foam combining experimental and numerical tools. Characterization tests have been conducted on Split Hopkinson Pressure Bars (SHPB) up to $5000 \mathrm{~s}^{-1}$ in unconfined and confined configurations. Ballistic tests on clamped aluminium target with spheres (small steel and bigger aluminium) evaluate the dynamic damping of the foam using an ultrahigh speed camera and 3D DIC at the target back face to measure dynamic deformation and evaluate the dynamic damping capability of the polymeric foam. Numerical simulations have been performed with promising results.

The authors thank THIOT-INGENIERIE laboratory team for performing all the tests and DGA Land Systems for funding of the experimental study.

\section{References}

1. H. Abdulhamid and Al., Characterization of ballistic response of an armor ceramic material: experimental tests and numerical modelling, Light Weight Armour Group Conference, Grenoble, France, (2016)

2. H. Abulhamid and Al., Study of the ballistic behaviour of UHMWPE composite material: experimental characterization and numerical simulation, 12th International Conference on the Mechanical and Physical Behaviour of Materials under Dynamic Loading, EPJ. Web. of Conf., 183, Arcachon, France, (2018)

3. J. Mespoulet and Al., Dynamic damping capability of a ballistic foam from characterization to numerical investigation, Light Weight Armour Group Conference, Grenoble, France, (2016) 